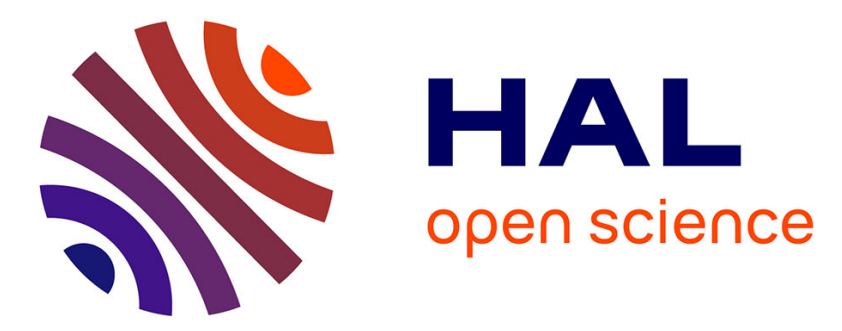

\title{
CRB derivation and new Code-Aided timing recovery technique for QAM modulated signals
}

Imen Nasr, Leila Najjar Atallah, Benoit Geller, Sofiane Cherif

\section{To cite this version:}

Imen Nasr, Leila Najjar Atallah, Benoit Geller, Sofiane Cherif. CRB derivation and new CodeAided timing recovery technique for QAM modulated signals. IEEE International Conference on Communications (ICC), 2015, Londres, United Kingdom. 10.1109/ICC.2015.7249099 hal-01225820

\section{HAL Id: hal-01225820 \\ https://hal.science/hal-01225820}

Submitted on 6 Dec 2015

HAL is a multi-disciplinary open access archive for the deposit and dissemination of scientific research documents, whether they are published or not. The documents may come from teaching and research institutions in France or abroad, or from public or private research centers.
L'archive ouverte pluridisciplinaire HAL, est destinée au dépôt et à la diffusion de documents scientifiques de niveau recherche, publiés ou non, émanant des établissements d'enseignement et de recherche français ou étrangers, des laboratoires publics ou privés. 


\title{
CRB Derivation and New Code-Aided Timing Recovery Technique for QAM Modulated Signals
}

\author{
Imen Nasr ${ }^{1,2}$, Leïla Najjar Atallah ${ }^{1}$, Benoît Geller ${ }^{2}$ and Sofiane Cherif ${ }^{1}$ \\ ${ }^{1}$ COSIM Research Lab, Higher School of Communications of Tunis, University of Carthage, Tunisia \\ ${ }^{2}$ U2IS Research Lab, Ecole Nationale Supérieure de Techniques Avancées ENSTA ParisTech, France \\ ${ }^{1}$ \{nasr.imen,leila.najjar,sofiane.cherif\}@ supcom.rnu.tn \\ ${ }_{2}^{2}$ imen.nasr,benoit.geller $\}$ ensta-paristech.fr
}

\begin{abstract}
In this paper, we propose a maximum likelihood based Code-Aided (CA) timing recovery algorithm for square-QAM modulated signals. We also theoretically derive the analytical expression of the CA Cramer-Rao Bound for time delay estimation. Our simulations show that the proposed CA approach realizes a performance equivalent to the Data-Aided (DA) approach over a large interval of signal to noise ratio (SNR) values.
\end{abstract}

\section{INTRODUCTION}

High spectrum efficiency modulations are required in modern communication systems in order to transmit high data rate information over a limited bandwidth [1]. This is why quadrature amplitude modulation (QAM) is commonly used. This modulation requires fine synchronization which is at the origin of the development of many time delay estimators. Some DA and non-data-aided (NDA) time delay estimation techniques have been employed in real systems for decades such as [2] and [3]. In DA estimation, reference signals are introduced in the transmitted frame to assist the synchronization process. Even if this technique performs well, it reduces the whole system throughput; this is avoided in the NDA mode where only useful information data, unknown to the receiver, is transmitted. This operating mode optimizes spectral efficiency, but NDA synchronization has low performance compared to the DA mode.

Nevertheless, with the development of channel coding techniques [4]-[7], iterative decoding has recently been extended to other receiver functions, especially time synchronization. Observing that turbo receivers usually operate at low SNR values, many researches have focused on CA synchronization algorithms [8] which consist in using the available soft information provided by the turbo detector to assist the synchronizer. This idea has already been applied in a number of contributions. For instance, it was applied for phase estimation in turbo decoding receivers [9]. It was also exploited in [10] with an expectation maximization algorithm in the Maximum Likelihood (ML) synchronization framework. Moreover, the timing synchronizer and the channel decoder can improve each other progressively by exchanging information using iterative techniques [11]. In [12] the authors have proposed to introduce the timing recovery inside the turbo equalizer to

${ }^{*}$ This work has been supported by the Greencocom project of the ANR jointly perform these tasks. In [13], an iterative timing recovery technique where the soft information from the decoder is fed into the Mueller and Muller (M\&M) timing error detector (TED) [2] is jointly performed with turbo equalization. A soft maximum likelihood timing recovery technique has also been proposed in [14] where a priori information on the transmitted symbols is used at the demapper and is injected to the M\&M TED.

The variance of the timing error is usually compared to a theoretical bound corresponding to the optimum performance. The Cramer-Rao Bound (CRB) is one of the most commonly used lower bounds on variance relative to the class of unbiased estimators and is the easiest to derive [15]. Closed-form expressions of the CRB for CA synchronization parameter estimation were specifically derived for carrier phase and frequency estimation. In [16]-[18], the authors derived the Bayesian and hybrid Cramer-Rao bounds (BCRB and HCRB) for the CA, the DA, and the NDA dynamical phase estimation of QAM modulated signals and theoretically showed the possible improvement using a CA technique. For timing recovery, only closed-form expressions of the DA CRB [19] and the NDA CRB for linearly modulated signal [20] have been derived. The modified CRB (MCRB) is easier to derive and averages the bound calculus over nuisance parameters, which in our case correspond to unknown symbols; the MCRB has been presented in [21], [22], however, it is looser and moves away from the true CRB, especially for low SNR.

Differently from [19], [20], the goal and contribution of this paper is to propose a CA timing recovery technique and a closed-form expression of the CRB for CA delay estimation in the case of square-QAM modulated signals.

This paper is organized as follows. In section II, the system model and the CA time delay estimation based on the ML approach are presented. In section III, the CA time synchronization algorithm is proposed. In section IV, closedform expressions of the CRB relative to CA mode are derived. Simulation results are provided in section $\mathrm{V}$ and validate our analysis. The last section concludes our work.

\section{SYSTEM MODEL}

Let us consider the linearly modulated transmitted signal $s(t)$ written as:

$$
s(t)=\sum_{i} a_{i} h(t-i T),
$$


where $a_{i}$ denotes the zero mean i.i.d. transmitted symbols drawn from a square-QAM constellation, $h(t)$ is the impulse response of the transmission filter and $T$ is the symbol period.

The received signal is:

$$
r(t)=s(t-\tau)+n(t),
$$

where $\tau$ is an unknown delay introduced by the channel to the transmitted signal $s(t)$ and $n(t)$ is an additive white Gaussian noise (AWGN) of zero mean and of variance $\sigma^{2}$. The maximum likelihood estimator of the time delay is given by the following equation [23]:

$$
\hat{\tau}=\arg \max _{u}\{\Lambda(u, \mathbf{a})\},
$$

where $\mathbf{a}$ is the vector of the transmitted symbols,

$$
\Lambda(u, \mathbf{a})=\exp \left(-\frac{1}{2 \sigma^{2}} \int_{T_{0}}|r(t)-s(t-u)|^{2} d t\right),
$$

is the likelihood function and $T_{0}$ is the observation interval We can equivalently use the log-likelihood function $\Lambda_{L}(u, \mathbf{a})$ instead of $\Lambda(u, \mathbf{a})$. Thus from (1)-(4) the time delay is estimated in the maximum likelihood sense according to:

$$
\hat{\tau}=\arg \max _{u}\left\{\Lambda_{L}(u, \mathbf{a})\right\},
$$

where:

$$
\Lambda_{L}(u, \mathbf{a})=\sum_{k}\left(\frac{\Re\left\{a_{k}^{*} x_{k}(u)\right\}}{\sigma^{2}}-\frac{\left|a_{k}\right|^{2}}{2 \sigma^{2}}\right),
$$

and

$$
\begin{aligned}
x_{k}(u) & =y_{k}(u)+v_{k}(u), \\
y_{k}(u) & =\sum_{i} a_{i} g((k-i) T-(\tau-u)), \\
v_{k}(u) & =\int_{T_{0}} h^{*}(t-k T-u) n(t) d t, \\
g(t) & =h \otimes h_{-}^{*}(t),
\end{aligned}
$$

where $\otimes$ denotes the convolution operation, $\Re\{z\}$ and $\Im\{z\}$ are the real and imaginary parts of $z, z^{*}$ is the conjugate of $z$ and for any function $f, f_{-}(t)=f(-t)$.

In equations (7)-(10), $x_{k}(u)$ is the matched filter output of the received signal, $y_{k}(u)$ is its useful information part, whereas $v_{k}(u)$ is a colored gaussian noise of zero mean and variance $\sigma^{2}$ and $g(t)$ is classically assumed to be a Nyquist pulse.

Since it is difficult in practice to maximize $\Lambda_{L}(u, \mathbf{a})$ and to find $u$ such that $\frac{\partial \Lambda_{L}(u, \mathbf{a})}{\partial u}=0$, we propose to implement an adaptive algorithm whose main objective aims at minimizing the derivative of the log-likelihood function towards zero. The updating equation of the adaptive algorithm is given by:

$$
\hat{\tau}_{k}=\hat{\tau}_{k-1}+\mu e_{k}\left(a_{k}, \hat{\tau}_{k-1}\right),
$$

where $\mu$ is the step-size and $e_{k}\left(a_{k}, \hat{\tau}_{k-1}\right)$ is the updating error such that:

$$
e_{k}\left(a_{k}, \hat{\tau}_{k-1}\right)=\Re\left\{\left.a_{k}^{*} \frac{\partial x_{k}(\tau)}{\partial \tau}\right|_{\tau=\hat{\tau}_{k-1}}\right\} .
$$

Such approach is called the ML Detector (MLD) [21]. The stepsize can be optimized [24] [25] but this is beyond the scope of our paper.
According to equation (12), in order to estimate $\tau$ we need to know the transmitted symbols $a_{k}$. This can be made by sending pilot symbols in the case of a DA mode or by making possibly unreliable hard decisions at the receiver in the case of a NDA mode. We hereafter propose to use more reliable softinformation from the turbo-decoder block to derive a code aided estimate of the symbols $a_{k}$. Also, this optimizes the spectral efficiency as it avoids to send any non informative reference signals.

\section{Proposed Code Aided Timing Detector}

Based on the maximum likelihood approach we propose in the following paragraph to derive a code aided adaptive algorithm for square-QAM modulated signals.

\section{A. Decoder's soft output for Gray-coded constellation}

We assume the constellation to be Gray-coded taking values in the alphabet set $\mathcal{V}=\left\{v_{1}, v_{1}, \ldots, v_{M}\right\}$ where $M$ is the size of the constellation. The chosen $i^{\text {th }}$ Gray-coded constellation element $v_{i}$ and the $k^{t h}$ transmitted symbol $a_{k}$ are denoted as:

$$
\begin{aligned}
v_{i} & \Leftrightarrow c_{1}^{i} c_{2}^{i} \ldots c_{\log _{2}(M)}^{i}, \\
a_{k} & \Leftrightarrow b_{1}^{k} b_{2}^{k} \ldots b_{\log _{2}(M)}^{k},
\end{aligned}
$$

where $c_{j}^{i}$ (resp. $b_{j}^{k}$ ) corresponds to the $j^{\text {th }}$ binary information of $v_{i}$ (resp. $\left.a_{k}\right)$.

We can assume that the coded bits in a symbol are statistically independent by using a large-size interleaver [5], [26] so that:

$$
\begin{aligned}
P\left[a_{k}=v_{i}\right] & =P\left[b_{1}^{k}=c_{1}^{i}, b_{2}^{k}=c_{2}^{i}, \ldots, b_{\log _{2}(M)}^{k}=c_{\log _{2}(M)}^{i}\right] \\
& =\prod_{m=1}^{\log _{2}(M)} P\left[b_{m}^{k}=c_{m}^{i}\right]
\end{aligned}
$$

Let us consider, $\lambda_{m}^{k}$ the soft output of the decoder at any time index $k$ such that:

$$
\lambda_{m}^{k}=\ln \left(\frac{P\left[b_{m}^{k}=1\right]}{P\left[b_{m}^{k}=0\right]}\right), m=1, \ldots, \log _{2}(M) .
$$

The soft-information $\lambda_{m}^{k}$ is generally obtained after several decoding iterations by a soft decoder. A general expression of the $a$ priori probability of the coded bit $b_{m}^{k}$, considering that the symbol $v_{i}$ is sent at time $k$, is given by:

$$
P\left[b_{m}^{k}=c_{m}^{i}\right]=\frac{\exp \left(\left(2 c_{m}^{i}-1\right) \frac{\lambda_{m}^{k}}{2}\right)}{2 \cosh \left(\frac{\lambda_{m}^{k}}{2}\right)},
$$

where $c_{m}^{i} \in\{0,1\}$. In order to compute $P\left(a_{k}=\tilde{v}_{i}\right)$ we use a Gray-mapping technique for $2^{2 p}$-QAM modulated signals. According to (15) and (17), for independent symbol bits, the symbol probability is given by:

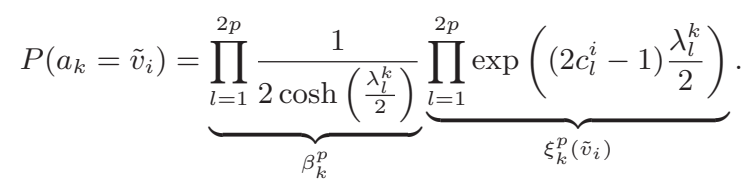

For notation convenience, we briefly describe the recurrence based construction of the Gray-mapping constellation starting 
from a $2^{2 p-2}-\mathrm{QAM}$ Gray coded constellation:

In the upper right quarter of the complex plan, $\forall \tilde{v}_{i} \in \tilde{\mathcal{V}}_{2^{2 p}}$ the $2 p-2$ most significant bits (MSB) are those of the symbols from $\mathcal{V}_{2^{2 p-2}}$. Then, $\forall \tilde{v}_{i} \in-\tilde{\mathcal{V}}_{2^{2 p}}$ the $2 p-2 \mathrm{MSB}$ are obtained by central point symmetry of those of $\tilde{\mathcal{V}}_{2^{2 p}}$. The $2 p-2$ MSB of the elements in $\tilde{\mathcal{V}}_{2^{2 p}}^{*}$ (resp. $-\tilde{\mathcal{V}}_{2^{2 p}}^{*}$ ) are obtained by symmetry on the $y$-axis (resp. $x$-axis) of those of $\tilde{\mathcal{V}}_{2^{2 p}}$.

Finally, the two least significant bits (LSB) of the the symbols from $\mathcal{V}_{2^{2 p}}$ are those of a Gray-coded QPSK constellation. In other terms:

$$
\begin{aligned}
\forall \tilde{v}_{i} \in \tilde{\mathcal{V}}_{2^{2 p}} & c_{2 p-1}^{i} c_{2 p}^{i}=11, \\
\forall \tilde{v}_{i} \in\left(-\tilde{\mathcal{V}}_{2^{2 p}}\right) & c_{2 p-1}^{i} c_{2 p}^{i}=00, \\
\forall \tilde{v}_{i} \in \tilde{\mathcal{V}}_{2^{2 p}}^{*} & c_{2 p-1}^{i} c_{2 p}^{i}=01, \\
\forall \tilde{v}_{i} \in\left(-\tilde{\mathcal{V}}_{2^{2 p}}^{*}\right) & c_{2 p-1}^{i} c_{2 p}^{i}=10 .
\end{aligned}
$$

The symbol probability (18) then becomes:

$$
\begin{aligned}
P\left(a_{k}=\tilde{v}_{i}\right)= & \beta_{k}^{p} \overbrace{\prod_{l=1}^{p-2} \exp \left(\left(2 c_{l}^{i}-1\right) \frac{\lambda_{l}^{k}}{2}\right)}^{\gamma_{k}^{p}\left(\tilde{v}_{i}\right)} \times \\
& \exp \left(\left(2 c_{2 p}^{i}-1\right) \frac{\lambda_{2 p}^{k}}{2}+\left(2 c_{2 p-1}^{i}-1\right) \frac{\lambda_{2 p-1}^{k}}{2}\right)
\end{aligned}
$$

In (23), $\gamma_{k}^{p}\left(\tilde{v}_{i}\right)$ deals with the first $2 p-2$ most significant bits (MSB) of $\tilde{v}_{i} \Leftrightarrow c_{1}^{i} c_{2}^{i} \ldots c_{2 p-1}^{i} c_{2 p}^{i}$. Regarding the construction symmetry of the Gray-code $\tilde{v}_{i},-\tilde{v}_{i}, \tilde{v}_{i}^{*}$ and $-\tilde{v}_{i}^{*}$ have the same $2 p-2$ MSBs. Accordingly:

$$
\gamma_{p}^{k}\left(\tilde{v}_{i}\right)=\gamma_{k}^{p}\left(-\tilde{v}_{i}\right)=\gamma_{k}^{p}\left(\tilde{v}_{i}^{*}\right)=\gamma_{k}^{p}\left(-\tilde{v}_{i}^{*}\right)
$$

\section{B. ML based derivation of an adaptive CA delay estimation}

In this paragraph, we derive the adaptive $\mathrm{CA}$ algorithm equation in the case of a $2^{2 p}$-QAM modulated signal $(M=$ $2^{2 p}$ ) by exploiting the structure of the Gray-mapping technique and the general properties of the constellation alphabet. In fact, an $2^{2 p}$-QAM modulated signal takes values into the set $\mathcal{V}_{2^{2 p}}=\left\{v_{m}= \pm(2 i-1) d_{p} \pm j(2 n-1) d_{p} ; i, n=1,2, \ldots, 2^{p}\right\}$, where $d_{p}$ is the intersymbol distance and $j^{2}=-1$. Since we do not know $a_{k}$, the time delay estimate in the maximum likelihood sense is given by:

$$
\hat{\tau}=\arg \max _{u}\{\Lambda(u)\} .
$$

$\Lambda(u)$ is obtained by averaging the likelihood function $\Lambda(u, \mathbf{a})$ over the possible values of the transmitted symbols $a_{k}$. Thus, by utilizing the independence property between the coded symbols and averaging over the set of possible transmitted symbols we find that:

$$
\Lambda(u)=\prod_{k} \sum_{v_{i} \in \mathcal{V}_{2^{2 p}}} P\left[a_{k}=v_{i}\right] \exp \left(\frac{\Re\left\{v_{i}^{*} x_{k}(u)\right\}}{\sigma^{2}}-\frac{\left|v_{i}\right|^{2}}{2 \sigma^{2}}\right) \text {. }
$$

Let us consider $\tilde{\mathcal{V}}_{2^{2 p}}=\left\{\tilde{v}_{m}=(2 i-1) d_{p}+j(2 n-1) d_{p}, i, n=\right.$ $\left.1, \ldots, 2^{p}\right\}$, so $\mathcal{V}_{2^{2 p}}=\tilde{\mathcal{V}}_{2^{2 p}} \cup\left(-\tilde{\mathcal{V}}_{2^{2 p}}\right) \cup\left(\tilde{\mathcal{V}}_{2^{2 p}}\right)^{*} \cup\left(-\tilde{\mathcal{V}}_{2^{2 p}}^{*}\right)$.
Then, the likelihood function can be written as:

$$
\begin{aligned}
\Lambda(u) & =\prod_{k} \sum_{\tilde{v}_{i} \in \tilde{\mathcal{V}}_{2^{2} p}} P\left(a_{k}=\tilde{v}_{i}\right) \exp \left(\frac{\Re\left\{\tilde{v}_{i}^{*} x_{k}(u)\right\}}{\sigma^{2}}-\frac{\left|\tilde{v}_{i}\right|^{2}}{2 \sigma^{2}}\right) \\
& +P\left(a_{k}=-\tilde{v}_{i}\right) \exp \left(-\frac{\Re\left\{\tilde{v}_{i}^{*} x_{k}(u)\right\}}{\sigma^{2}}-\frac{\left|\tilde{v}_{i}\right|^{2}}{2 \sigma^{2}}\right) \\
& +P\left(a_{k}=\tilde{v}_{i}^{*}\right) \exp \left(\frac{\Re\left\{\tilde{v}_{i} x_{k}(u)\right\}}{\sigma^{2}}-\frac{\left|\tilde{v}_{i}\right|^{2}}{2 \sigma^{2}}\right) \\
& +P\left(a_{k}=-\tilde{v}_{i}^{*}\right) \exp \left(-\frac{\Re\left\{\tilde{v}_{i} x_{k}(u)\right\}}{\sigma^{2}}-\frac{\left|\tilde{v}_{i}\right|^{2}}{2 \sigma^{2}}\right) \cdot
\end{aligned}
$$

Replacing the symbol probability (23) into (27) yields the following expression of the likelihood function:

$$
\begin{aligned}
\Lambda(u)= & \prod_{k} \frac{2 \beta_{k}^{p}}{\sigma^{2}} \sum_{\tilde{v}_{i} \in \tilde{\mathcal{V}}_{2^{2 p}}} \exp \left(-\frac{\left|\tilde{v}_{i}\right|^{2}}{2 \sigma^{2}}\right) \gamma_{k}^{p}\left(\tilde{v}_{i}\right) \times \\
& {\left[\cosh \left(\frac{\Re\left\{\tilde{v}_{i}^{*} x_{k}(u)\right\}}{\sigma^{2}}+\frac{\lambda_{2 p-1}^{k}}{2}+\frac{\lambda_{2 p}^{k}}{2}\right)\right.} \\
& \left.\cosh \left(\frac{\Re\left\{\tilde{v}_{i} x_{k}(u)\right\}}{\sigma^{2}}-\frac{\lambda_{2 p-1}^{k}}{2}+\frac{\lambda_{2 p}^{k}}{2}\right)\right] .
\end{aligned}
$$

A similar development of the likelihood function has been made in [27] to compute the Cramer Rao Bound for the CA SNR estimation and it has been shown that:

$$
\gamma_{k}^{p}\left(\tilde{v}_{m}\right)=\theta_{k, 2 p}(i) \theta_{k, 2 p-1}(n),
$$

where $\tilde{v}_{m}= \pm(2 i-1) d_{p} \pm j(2 n-1) d_{p}$ and $\theta_{k, 2 p}(i)$ and $\theta_{k, 2 p-1}(n)$ are recursively obtained according to the following equations:

$$
\begin{aligned}
\theta_{k, 2 p}(i)= & \theta_{k, 2 p-2}\left(\frac{\left|2 i-1-2^{p-1}\right|+1}{2}\right) \times \\
& \exp \left(\left(2\left\lfloor\frac{i-1}{\left.\left.2^{p-2}\right\rfloor-1\right)} \frac{\lambda_{2 p-2}^{k}}{2}\right),\right.\right. \\
\theta_{k, 2 p-1}(n)= & \theta_{k, 2 p-3}\left(\frac{\left|2 n-1-2^{p-1}\right|+1}{2}\right) \times \\
& \exp \left(\left(2 \left\lfloor\frac{n-1}{\left.\left.\left.2^{p-2}\right\rfloor-1\right) \frac{\lambda_{2 p-3}^{k}}{2}\right),}\right.\right.\right.
\end{aligned}
$$

where $\lfloor x\rfloor$ is the integer part of $x, \theta_{k, 1}(1)=1$ and $\theta_{k, 2}(1)=1$.

Using the identity $\cosh (a)+\cosh (b)=$ $2 \cosh \left(\frac{a+b}{2}\right) \cosh \left(\frac{a-b}{2}\right)$, the likelihood function can then be written as:

$$
\Lambda(u)=\prod_{k} \frac{4 \beta_{k}^{p}}{\sigma^{2}} H_{k}^{2 p}\left(r_{k}(u)\right) H_{k}^{2 p-1}\left(i_{k}(u)\right),
$$

where

$$
\begin{aligned}
H_{k}^{l}(x)= & \sum_{i=1}^{2^{p-1}} \theta_{k, l}(i) \exp \left(-\frac{(2 i-1)^{2} d_{p}^{2}}{2 \sigma^{2}}\right) \times \\
& \cosh \left(\frac{(2 i-1) d_{p} x}{\sigma^{2}}+(-1)^{l} \frac{\lambda_{l}^{k}}{2}\right),
\end{aligned}
$$

$r_{k}(u)=\Re\left\{x_{k}(u)\right\}$ and $i_{k}(u)=\Im\left\{x_{k}(u)\right\}$. 
Then, the derivative of the log-likelihood function is:

$\frac{\partial \Lambda_{L}(u)}{\partial u}=\sum_{k} \frac{\dot{H}_{k}^{2 p}\left(r_{k}(u)\right)}{H_{k}^{2 p}\left(r_{k}(u)\right)} \frac{\partial r_{k}(u)}{\partial u}+\frac{\dot{H}_{k}^{2 p-1}\left(i_{k}(u)\right)}{H_{k}^{2 p-1}\left(i_{k}(u)\right)} \frac{\partial i_{k}(u)}{\partial u}$,

where for any function $f($.$) , we note \dot{f}(u)=\frac{\partial f(u)}{\partial u}$.

If we consider the expressions of the real and the imaginary parts of the soft symbol:

$$
\begin{aligned}
& \Re\left\{\tilde{a}_{k}\right\}=\frac{\dot{H}_{k}^{2 p}\left(r_{k}(u)\right)}{H_{k}^{2 p}\left(r_{k}(u)\right)} \\
& \Im\left\{\tilde{a}_{k}\right\}=\frac{\dot{H}_{k}^{2 p-1}\left(i_{k}(u)\right)}{H_{k}^{2 p-1}\left(i_{k}(u)\right)},
\end{aligned}
$$

then, we find that:

$$
\frac{\partial \Lambda_{L}(u)}{\partial u}=\sum_{k} \Re\left\{\tilde{a}_{k}^{*} \frac{\partial x_{k}(u)}{\partial u}\right\} .
$$

In practice, we propose to estimate $\tau$, using the updating equation (11) by substituting $a_{k}$ with the soft symbol $\tilde{a}_{k}$; the updating equation becomes:

$$
\hat{\tau}_{k}=\hat{\tau}_{k-1}+\mu e_{k}\left(\tilde{a}_{k}, \hat{\tau}_{k-1}\right),
$$

where the real and imaginary parts of $\tilde{a}_{k}$ are given by (34) and (35) for $u=\hat{\tau}_{k-1}$ and $e_{k}\left(\tilde{a}_{k}, \hat{\tau}_{k-1}\right)=\Re\left\{\left.\tilde{a}_{k}^{*} \frac{\partial x_{k}(\tau)}{\partial \tau}\right|_{\tau=\hat{\tau}_{k-1}}\right\}$.

The performance of these estimators can also be compared to the corresponding theoretical Cramer-Rao Bound. The analytical expression of this bound is evaluated in the next section for a code aided time synchronization algorithm.

\section{Cramer-Rao Bound}

In this section, we derive the Cramer-Rao Bound (CRB) for a code aided delay estimation in the case of square-QAM modulated signals.

Suppose that we are able to produce an unbiased estimate $\hat{\tau}$ of the delay $\tau$ from the received signal. Thus, the CRB which verifies $E\left[(\hat{\tau}-\tau)^{2}\right] \geq \operatorname{CRB}(\tau)$ for any unbiased estimator $\hat{\tau}$ of $\tau$ is defined as [15]:

$$
\operatorname{CRB}(\tau)=I^{-1}(\tau)
$$

where $I(\tau)$ is the Fisher information matrix (FIM) [15] expressed as:

$$
I(\tau)=E\left[\left(\frac{\partial \Lambda_{L}(\tau)}{\partial \tau}\right)^{2}\right]
$$

From (33) we have:

$$
\begin{aligned}
E\left[\left(\frac{\partial \Lambda_{L}(\tau)}{\partial \tau}\right)^{2}\right]= & \sum_{k} \sum_{n} \sum_{l \in\{2 p, 2 p-1\}} E\left[\frac{\dot{H}_{k}^{l}\left(z_{k, l}(\tau)\right)}{H_{k}^{l}\left(z_{k, l}(\tau)\right)} \times\right. \\
& \left.\frac{\dot{H}_{n}^{l}\left(z_{n, l}(\tau)\right)}{H_{n}^{l}\left(z_{n, l}(\tau)\right)} \frac{\partial z_{k, l}(\tau)}{\partial \tau} \frac{\partial z_{n, l}(\tau)}{\partial \tau}\right] \\
+ & \sum_{k} \sum_{n} E\left[\frac{\dot{H}_{k}^{2 p}\left(z_{k, 2 p}(\tau)\right)}{H_{k}^{2 p}\left(z_{k, 2 p}(\tau)\right)} \frac{\partial z_{k, 2 p}(\tau)}{\partial \tau}\right. \\
\times & \left.\frac{\dot{H}_{n}^{2 p-1}\left(z_{n, 2 p-1}(\tau)\right)}{H_{n}^{2 p-1}\left(z_{n, 2 p-1}(\tau)\right)} \frac{\partial z_{n, 2 p-1}(\tau)}{\partial \tau}\right],
\end{aligned}
$$

where

$$
z_{k, l}(\tau)=\left\{\begin{array}{l}
r_{k}(\tau) \text { if } l=2 p \\
i_{k}(\tau) \text { if } l=2 p-1
\end{array}\right.
$$

We note that $\frac{\partial z_{k, 2 p}(\tau)}{\partial \tau}$ (resp. $\frac{\partial z_{k, 2 p-1}(\tau)}{\partial \tau}$ ) and $z_{k, 2 p}(\tau)$ (resp. $\left.z_{k, 2 p-1}(\tau)\right)$ are uncorrelated (see [20]), therefore the second term of (40) is equal to 0 . Deriving the CRB then resorts to compute the first term of (40), which will be carried hereafter distinguishing the cases $k=n$ and $k \neq n$. Case 1: $k=n$ :

$$
\begin{aligned}
& E\left[\left(\frac{\dot{H}_{k}^{l}\left(z_{k, l}(\tau)\right)}{H_{k}^{l}\left(z_{k, l}(\tau)\right)}\right)^{2}\left(\frac{\partial z_{k, l}(\tau)}{\partial \tau}\right)^{2}\right] \\
= & E\left[\left(\frac{\dot{H}_{k}^{l}\left(z_{k, l}(\tau)\right)}{H_{k}^{l}\left(z_{k, l}(\tau)\right)}\right)^{2}\right] E\left[\left(\frac{\partial z_{k, l}(\tau)}{\partial \tau}\right)^{2}\right] .
\end{aligned}
$$

On the other hand,

$$
E\left[\left(\frac{\partial z_{k, l}(\tau)}{\partial \tau}\right)^{2}\right]=\sum_{i} E\left[a_{i}^{2}\right] \dot{g}^{2}((k-i) T)-\frac{\sigma^{2}}{2} \ddot{g}(0),
$$

where for any function $f$, we note $\ddot{f}(\tau)=\frac{\partial^{2} f(\tau)}{\partial \tau^{2}}$. We have:

$E\left[\left(\frac{\dot{H}_{k}^{l}\left(z_{k, l}(\tau)\right)}{H_{k}^{l}\left(z_{k, l}(\tau)\right)}\right)^{2}\right]=\int_{-\infty}^{+\infty}\left(\frac{\dot{H}_{k}^{l}\left(z_{k, l}(\tau)\right)}{H_{k}^{l}\left(z_{k, l}(\tau)\right)}\right)^{2} P\left(z_{k, l}(\tau)\right) d z_{k, l}(\tau)$.

$g$ being a Nyquist filter, the received matched filtered signal can be written as:

$$
x_{k}(\tau)=a_{k}+v_{k}(\tau),
$$

so that from (23) and (45):

$$
\begin{aligned}
P\left(x_{k}(\tau)\right)= & \sum_{v_{i} \in \mathcal{V}} \exp \left(-\frac{\left|x_{k}(\tau)-v_{i}\right|^{2}}{2 \sigma^{2}}\right) \frac{P\left(a_{k}=v_{i}\right)}{2 \pi \sigma^{2}} \\
= & \frac{4 \beta_{k}}{2 \pi \sigma^{2}} H_{k}^{2 p}\left(r_{k}(\tau)\right) H_{k}^{2 p-1}\left(i_{k}(\tau)\right) \times \\
& \exp \left(-\frac{r_{k}^{2}(\tau)+i_{k}^{2}(\tau)}{2 \sigma^{2}}\right) .
\end{aligned}
$$

Given that $r_{k}(\tau)$ and $i_{k}(\tau)$ are respectively the real and imaginary parts of $x_{k}(\tau)$, which are two independent random variables identically distributed, therefore:

$$
P\left(x_{k}(\tau)\right)=P\left(r_{k}(\tau)\right) P\left(i_{k}(\tau)\right)
$$

where

$$
\begin{aligned}
P\left(r_{k}(\tau)\right) & =\frac{2 \omega_{k, 2 p}}{\sqrt{2 \pi \sigma^{2}}} H_{k}^{2 p}\left(r_{k}(\tau)\right) \exp \left(-\frac{r_{k}^{2}(\tau)}{2 \sigma^{2}}\right) \\
P\left(i_{k}(\tau)\right) & =\frac{2 \omega_{k, 2 p-1}}{\sqrt{2 \pi \sigma^{2}}} H_{k}^{2 p-1}\left(i_{k}(\tau)\right) \exp \left(-\frac{i_{k}^{2}(\tau)}{2 \sigma^{2}} \gamma_{49)}\right.
\end{aligned}
$$

and

$$
\begin{aligned}
\omega_{k, 2 p} & =\prod_{l=1}^{p} \frac{1}{2 \cosh \left(\frac{\lambda_{2 l}^{k}}{2}\right)} \\
\omega_{k, 2 p-1} & =\prod_{l=1}^{p} \frac{1}{2 \cosh \left(\frac{\lambda_{2 l-1}^{k}}{2}\right)}
\end{aligned}
$$


As a result we obtain:

$E\left[\left(\frac{\dot{H}_{k}^{l}\left(z_{k, l}(\tau)\right)}{H_{k}^{l}\left(z_{k, l}(\tau)\right)}\right)^{2}\right]=\frac{2 \omega_{k, l}}{\sqrt{2 \pi \sigma^{2}}} \int_{-\infty}^{+\infty} \frac{\dot{H}_{k}^{l}(x)^{2}}{H_{k}^{l}(x)} \exp \left(-\frac{x^{2}}{2 \sigma^{2}}\right) d x$.

Case 2: $k \neq n$ :

Given that $\frac{\partial z_{k, l}(\tau)}{\partial \tau}$ and $\frac{\partial z_{n, l}(\tau)}{\partial \tau}$ are dependent on $z_{k, l}(\tau)$ and $z_{n, l}(\tau)$, in order to derive the desired expectation in the first term of (40), we first average by conditioning on $z_{k, l k}(\tau)$ and $z_{n, l}(\tau)$, then we average the resulting expression with respect to these two random variables.

We have:

$$
z_{k, l}(\tau)=a_{k, l}+\alpha_{k, l}
$$

where $a_{k, l}$ (resp. $\alpha_{k, l}$ ) is equal to $\Re\left\{a_{k}\right\}$ (resp. $\Re\left\{v_{k}(\tau)\right\}$ ) if $l=2 p$ and to $\Im\left\{a_{k}\right\}$ (resp. $\Im\left\{v_{k}(\tau)\right\}$ ) if $l=2 p-1$.

$$
\begin{aligned}
\frac{\partial z_{n, l}(\tau)}{\partial \tau}= & a_{k, l} \dot{g}((n-k) T)+\sum_{j, j \neq k, n} a_{n} \dot{g}((n-j) T)+\dot{\alpha}_{n, l} \\
= & \left(z_{k, l}(\tau) \dot{g}((n-k) T)-\dot{g}((n-k) T) \alpha k, l\right) \\
& +\sum_{j, j \neq k, n} a_{j, l} \dot{g}((n-j) T)+\dot{\alpha}_{n, l}
\end{aligned}
$$

Evaluating a conditional mean of (54) we then find that:

$$
\begin{aligned}
& E\left[\frac{\partial z_{n, l}(\tau)}{\partial \tau} \times \frac{\partial z_{k, l}(\tau)}{\partial \tau} \mid z_{k, l}(\tau), z_{n, l}(\tau)\right] \\
= & z_{k, l}(\tau) \dot{g}((n-k) T) z_{n, l}(\tau) \dot{g}((k-n) T) \\
= & -z_{k, l}(\tau) x_{n, l}(\tau) \dot{g}((k-n) T)^{2},
\end{aligned}
$$

so that:

$$
\begin{aligned}
& E\left[\frac{\partial z_{n, l}(\tau)}{\partial \tau} \frac{\partial z_{k, l}(\tau)}{\partial \tau} \frac{\dot{H}_{k}^{l}\left(z_{k, l}(\tau)\right)}{H_{k}^{l}\left(z_{k, l}(\tau)\right)} \times\right. \\
& \left.\frac{\dot{H}_{n}^{l}\left(z_{n, l}(\tau)\right)}{H_{n}^{l}\left(z_{n, l}(\tau)\right)} \mid z_{k, l}(\tau), z_{n, l}(\tau)\right] \\
= & \left.\left.-\frac{\dot{H}_{k}^{l}\left(z_{k, l}(\tau)\right)}{H_{k}^{l}\left(z_{k, l}(\tau)\right)} \frac{\dot{H}_{n}^{l}\left(z_{n, l}(\tau)\right)}{H_{n}^{l}\left(z_{n, l}(\tau)\right)} z_{k, l}(\tau)\right) z_{n, l}(\tau)\right) \dot{g}((k-n) T)^{2} .
\end{aligned}
$$

(56) Fig. 1. MSE vs SNR for 16QAM modulated signal

Finally, we obtain:

$$
\begin{aligned}
& E\left[\frac{\partial z_{n, l}(\tau)}{\partial \tau} \frac{\partial z_{k, l}(\tau)}{\partial \tau} \frac{\dot{H}_{k}^{l}\left(z_{k, l}(\tau)\right)}{H_{k}^{l}\left(z_{k, l}(\tau)\right)} \frac{\dot{H}_{n}^{l}\left(z_{n, l}(\tau)\right)}{H_{n}^{l}\left(z_{n, l}(\tau)\right)}\right] \\
= & \left.\left.-E\left[z_{k, l}(\tau)\right) \frac{\dot{H}_{k}^{l}\left(z_{k, l}(\tau)\right)}{H_{k}^{l}\left(z_{k, l}(\tau)\right)}\right] E\left[z_{n, l}(\tau)\right) \frac{\dot{H}_{n}^{l}\left(z_{n, l}(\tau)\right)}{H_{n}^{l}\left(z_{n, l}(\tau)\right)}\right] \times \\
& \dot{g}((k-n) T)^{2} \\
= & \left.-E\left[z_{k, l}(\tau)\right) \frac{\dot{H}_{k}^{l}\left(z_{k, l}(\tau)\right)}{H_{k}^{l}\left(z_{k, l}(\tau)\right)}\right]^{2} \dot{g}((k-n) T)^{2}
\end{aligned}
$$

where

$E\left[z_{k, l}(\tau) \frac{\dot{H}_{k}^{l}\left(z_{k, l}(\tau)\right)}{H_{k}^{l}\left(z_{k, l}(\tau)\right)}\right]=\frac{2 \omega_{k, l}}{\sqrt{2 \pi \sigma^{2}}} \int_{-\infty}^{+\infty} x \dot{H}_{k}^{l}(x) \exp \left(-\frac{x^{2}}{2 \sigma^{2}}\right) d x$.

We mention that the integrand functions involved in (52) and (58), respectively, decrease rapidly as $x$ increases. Therefore, the integrals over $[-\infty,+\infty]$ can be accurately approximated by a finite integral over an interval $[-A,+A]$ and the Riemann integration method can be adequately used. The evaluation of the CRB is thus possible as all implied expressions in equation (40) are derived. Results will be displayed in the next section.

\section{Simulation Results}

In this section, we display the simulation results of the proposed CA time delay estimation algorithms and the proposed expression of the CRB. We consider the case of 16-QAM and 64-QAM signals with an up-sampling factor equal to 8 , passed through a raised cosine filter, with a roll-off factor $\delta$. Results are given for 200 symbol blocks averaged over 1000 Monte Carlo iterations. The LLRs are provided from the encoder which is composed of two identical RSCs concatenated in parallel with systematic rate $r=\frac{1}{2}$ and generator polynomials $(1,0,1,1)$ and $(1,1,0,1)$. A large interleaver is placed between the two RSCs. $\hat{\tau}_{k}$ is initialized to 0 and its estimated value is depicted at the end of the block when the steady state is achieved. The value of $x_{k}\left(\tau_{k-1}\right)$ is obtained via a quadratic interpolation.
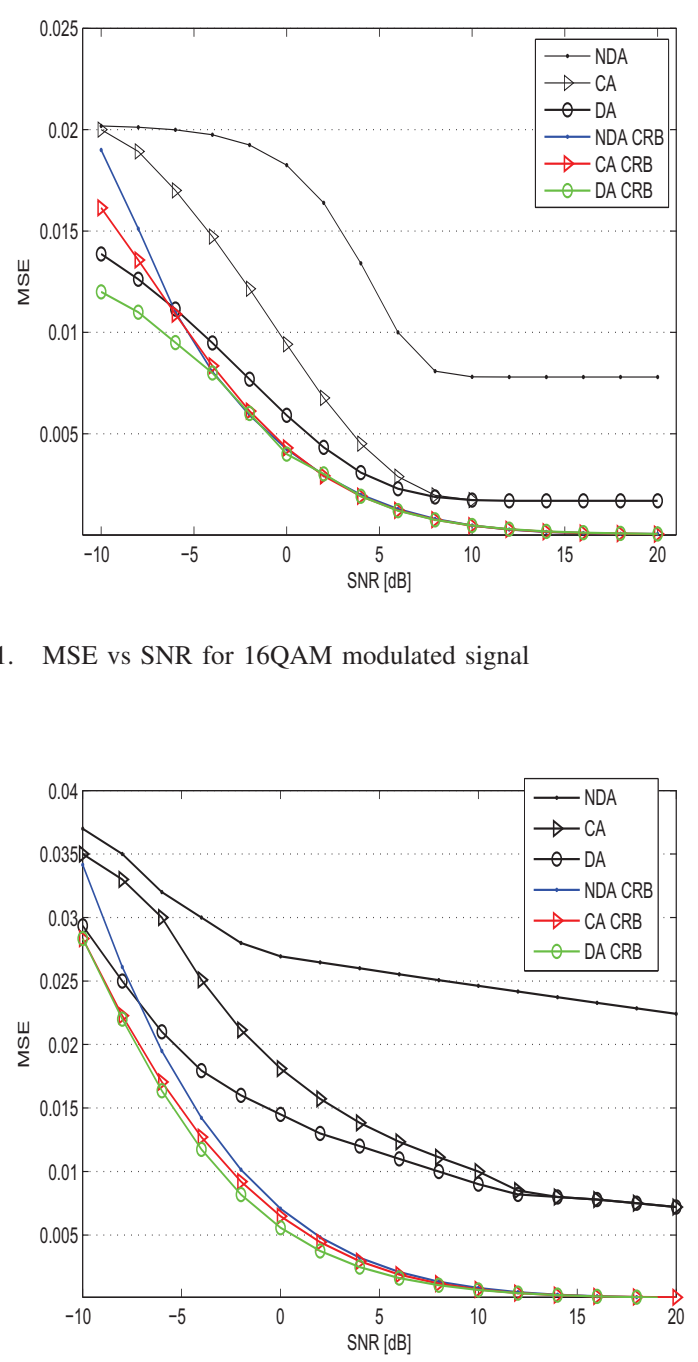

Fig. 2. MSE vs SNR for 64QAM modulated signal

In Fig. 1 and 2, we display simulation results of the mean square error (MSE) on $(\tau / T)$ as a function of the SNR for 16-QAM and 64-QAM respectively. For each figure, one can compare the estimation performance of the DA, CA and NDA mode. Compared to the NDA mode, the MSE is decreased by 
exploiting the soft-information from the turbo-decoder. The DA mode still achieves the best performance, however, it leads to a higher loss of spectral efficiency. Besides, over a wide range of SNRs, the CA mode performance is almost equivalent to that of the DA mode with no need for pilot symbols. For instance, for an $\mathrm{SNR}=0 \mathrm{~dB}$ the MSE is equal to $6.10^{-3}$ for the DA mode, $9.10^{-3}$ for the proposed technique and $1.8 \cdot 10^{-2}$ for the NDA mode for 16-QAM signals and it is equal to $1.5 \cdot 10^{-2}$ for the DA mode, $1.8 \cdot 10^{-2}$ for the proposed technique and $2.7 .10^{-2}$ for the NDA mode for 64QAM signals. The saturation of the MSE at the right side of Fig. 1 and 2 is due to the self noise of the updating error (12). Similar results are obtained for other roll-off factors.

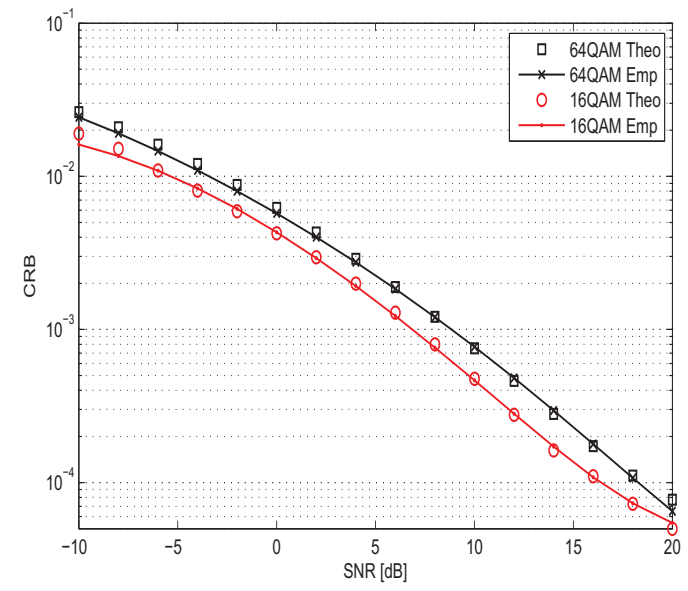

Fig. 3. Comparison between the empirical CRB and the analytical expression for different modulations, $\delta=0.3$

Fig. 3 depicts the evaluated theoretical expression of the $\mathrm{CRB}$ and the empirical CRB obtained by an averaging over constellation symbols through a Monte Carlo simulation for both 16-QAM and 64-QAM signals. We can confirm that the analytical values perfectly match the simulated values of the CRB thus assessing the validity of the derived CRB.

\section{CONCLUSION}

In this paper, we proposed a new code aided estimation algorithm for time delay recovery of square QAM modulated signals. The proposed CA algorithm performs better than the NDA algorithm and reaches the performance of the DA one over a large interval of SNR values with no need for reference signals. We also established the analytical expression of the CRB for code aided time delay estimation for square-QAM signals. This expression matched the empirical results obtained by simulation. Some future works aim at transposing our approach to the Bayesian canvas [28].

\section{REFERENCES}

[1] L. Zhou, B. Geller, A. Wei, B. Zheng, J. Cui, and S. Xu, "Crosslayer rate allocation for multimedia applications in pervasive computing environment," in IEEE GLOBECOM, Nov 2008, pp. 1-5.

[2] K. Mueller and M. Muller, "Timing recovery in digital synchronous data receivers," IEEE Trans. on Communications, vol. 24, no. 5, pp. 516-531, 1976.
[3] F. M. Gardner, "A bpsk/qpsk timing-error detector for sampled receivers," IEEE Trans. on Communications, vol. 34, no. 5, pp. 423-429, 1986.

[4] R. Gallager, "Low-density parity-check codes," IEEE Trans. on Information Theory, vol. 8, no. 1, pp. 21-28, Jan. 1962.

5] C. Berrou and A. Glavieux, "Near optimum error correcting coding and decoding: turbo-codes," IEEE Trans. on Communications, vol. 44 no. 10 , pp. 1261-1271, Oct. 1996.

[6] C. Vanstraceele, B. Geller, J.-P. Barbot, and J. M. Brossier, "Block turbo codes for multicarrier local loop transmission," vol. 3, pp. 1773-1776 vol.3, 2002.

[7] C. Vanstraceele, B. Geller, J. M. Brossier, and J.-P. Barbot, "A low complexity block turbo decoder architecture - [transactions letters]," IEEE Trans. on Communications, vol. 56, no. 12, pp. 1985-1987, December 2008.

[8] C. Berrou, J. Hagenauer, M. Luise, L. Vandendorpe, and C. Schegel, "Turbo-Information processing: algorithms, implementations \& applications," Proc. of the IEEE, vol. 95, no. 6, pp. 1146 - 1149, Jun. 2007.

[9] V. Lottici and M. Luise, "Carrier phase recovery for turbo-coded linear modulations," IEEE ICC, vol. 3, pp. 1541-1545, 2002.

[10] N. Noels, C. Herzet, A. Dejonghe, V. Lottici, H. Steendam, M. Moeneclaey, M. Luise, and L. Vandendorpe, "Turbo synchronization: an em algorithm interpretation,” IEEE ICC, vol. 4, pp. 2933-2937, 2003.

[11] L. Zhang and A. Burr, "APPA symbol timing recovery scheme for turbo codes," IEEE PIMRC, vol. 1, pp. 44-48, Sep. 2002.

[12] A. Nayak, J. Barry, and S. McLaughlin, "Joint timing recovery and turbo equalization for coded partial response channels," IEEE Trans. on Magnetics, vol. 38, no. 5, pp. 2295-2297, Sep. 2002.

[13] R. Barry, A. Kavcic, S. McLaughlin, A. Nayak, and W. Zeng, "Iterative timing recovery," IEEE Signal Processing Magazine, vol. 21, no. 1, pp. 89-102, Jan. 2004.

[14] I. Nasr, L. Najjar Atallah, S. Cherif, B. Geller, and J. Yang, "A soft maximum likelihood technique for time delay recovery," IEEE COMNET, Mar. 2014.

[15] S. M. Kay, Fundamentals of Statistical Signal Processing: Estimation Theory. Upper Saddle River, NJ, USA: Prentice-Hall, Inc., 1993.

[16] J. Yang, B. Geller, and S. Bay, "Bayesian and hybrid cramer-rao bounds for the carrier recovery under dynamic phase uncertain channels," IEEE Trans. on Signal Processing, vol. 59, no. 2, pp. 667-680, Feb 2011.

[17] S. Bay, B. Geller, A. Renaux, J. P. Barbot, and J. M. Brossier, "On the hybrid cramer-rao bound and its application to dynamical phase estimation," IEEE Signal Processing letters, vol. 15, pp. 453-456, 2008.

[18] S. Bay, C. Herzet, J. M. Brossier, J. P. Barbot, and B. Geller, "Analytic and asymptotic analysis of bayesian cramer-rao bound for dynamical phase offset estimation,' IEEE Trans. on Signal Processing, vol. 56, no. 1, pp. 61-70, Jan. 2008.

[19] H. Meyr, M. Moeneclaey, and S. A. Fechtel, Digital Communication Receivers, J. G. Proakis Series, Ed. Wiley Series in Telecommunications and Signal Processing, 1998.

[20] A. Masmoudi, F. Bellili, S. Affes, and A. Stephenne, "Closed-form expressions for the exact cramer-rao bounds of timing recovery estimators from bpsk, msk and square-qam transmissions," IEEE Trans. on Signal Processing, vol. 59, no. 6, pp. 2474-2484, Jun. 2011.

[21] U. Mengali and A. N. D'Andrea, Synchronization Techniques for Digital Receivers. Plenum Press, New York and London, 1997.

[22] A. D'Andrea, U. Mengali, and R. Reggiannini, "The modified cramerrao bound and its application to synchronization problems," IEEE Trans. on Communications, vol. 42, no. 234, pp. 1391-1399, Feb. 1994.

[23] M. Oerder, "Derivation of gardner's timing-error detector from the maximum likelihood principle," IEEE Trans. on Communications, vol. 35, no. 6, pp. 684-685, 1987.

[24] B. Geller, V. Capellano, J. M. Brossier, A. Essebbar, and G. Jourdain, "Equalizer for video rate transmission in multipath underwater communications," IEEE Journal of Oceanic Engineering, vol. 21, no. 2, pp. 150-156, Apr. 1996.

[25] J. Yang and B. Geller, "Near-optimum low-complexity smoothing loops for dynamical phase estimation," IEEE Trans. on Signal Processing, vol. 57, no. 9, pp. 3704-3711, Sep 2009.

[26] B. Vucetic and J. Yuan, Turbo Codes: Principles and Applications. Norwell, MA, USA: Kluwer Academic Publishers, 2000.

[27] F. Bellili, A. Methenni, and S. Affes, "Closed-form cramer rao lower bound for snr estimation from turbo-coded bpsk-, msk-, and square-qammodulated signals," IEEE Trans. on Signal Processing, vol. 62, no. 15, pp. 4018-4033, Aug. 2014.

[28] J. M. Brossier, P. O. Amblard, and B. Geller, "Self adaptive PLL for general qam constellations," Proc. of EUSIPCO, pp. 631-635, Sep. 2002. 